Reprod. Nutr. Dévelop., 1987, 27 (1 B), 241-242.

\title{
Distribution des bactéries adhérentes \\ en fonction de la taille des particules dans les contenus du rumen et du feuillet chez la vache laitière
}

D. BAUCHART, Françoise LEGAY-CARMIER, M. DOREAU $\left({ }^{*}\right)$

Laboratoire d'Etude du Métabolisme énergétique

(*) Laboratoire de la Lactation

I.N.R.A. Theix, 63122 Ceyrat, France.

Summary. The distribution of solid-adherent bacteria (SAB) in the solid fraction of rumen and omasum mainly was related to particle size. The mean concentrations of $S A B$ in total particles were similar in the rumen $(5.1 \pm 0.8 \%$ of DM) and the omasum $(5.4 \pm 0.1 \%$ of DM). The longest particles $(\geq 4 \mathrm{~mm})$ in the rumen and the smallest particles $(<0.1 \mathrm{~mm})$ in the omasum, which were the major classes, presented similar SAB contents $(6.4$ and $6.8 \%$ of DM). This suggested that the fixation rate of SAB was not affected by particle size.

La détermination du flux bactérien duodénal repose sur la mesure du flux de bactéries associées à la phase liquide (BAL) bien que celles-ci soient très minoritaires dans le rumen $(20 \%)$ par rapport aux bactéries adhérant à la phase solide (BAS) $(80 \%)$ (Bauchart et al., 1986). De plus, leur composition chimique étant très différente (Merry et Mc Allan, 1983), il importe de déterminer leur part respective dans le contenu quittant le rumen. La réduction en taille des particules dans ce contenu pouvant modifier le taux de fixation des bactéries, nous avons comparé chez la vache laitière la concentration des bactéries BAS suivant la taille des particules dans le rumen et le feuillet.

Matériel et méthodes. Les contenus de rumen et de feuillet ont été prélevés sur 4 vaches Pie Noires abattues le matin 2 h après avoir reçu en quantités égales du foin de fétuque et un aliment concentré.

La séparation selon leur taille des particules des contenus a été effectuée par tamisage en milieu liquide sur 5 tamis à maille d'ouverture décroissant de 4 à $0,1 \mathrm{~mm}$. Les bactéries fixées sur chaque classe de particules ont été extraites par battage au " stomacher 》 et purifiées par centrifugation (Bauchart et al., 1986). Les quantités de particules et de bactéries ont été déterminées par gravimétrie après séchage à l'étuve à $80{ }^{\circ} \mathrm{C}$ pendant $48 \mathrm{~h}$ et après lyophilisation respectivement.

Résultats et discussion. Pour le régime étudié, les particules alimentaires supérieures à $4 \mathrm{~mm}$ représentent $51,3 \%$ de la MS totale des particules du rumen et $3,5 \%$ de celle du feuillet, et les particules inférieures à $0,1 \mathrm{~mm} 24,5 \%$ et $41,6 \%$ de la MS. Les 4 autres classes représentent chacune en moyenne 6,1\% de la MS des particules dans le rumen et $13,7 \%$ dans le feuillet (tabl. 1 ).

La répartition des bactéries totales fixées suit sensiblement celle des particules : dans le rumen, $61,6 \%$ des bactéries sont fixés sur les particules de plus de $4 \mathrm{~mm}$, et dans le feuillet $52,6 \%$ sur celles inférieures à $0,1 \mathrm{~mm}$ (tabl. 1).

La concentration moyenne en poids des bactéries totales fixées sur. les particules est comparable dans le rumen $(5,1 \pm 0,8 \%$ de la MS des particules) et dans le feuillet $(5,4 \pm 0,1 \%)$. Cependant, les concentrations en bactéries sont 
significativement différentes $(P<0,05)$ dans le rumen et le feuillet pour les particules supérieures à $4 \mathrm{~mm}$ et inférieures à $0,1 \mathrm{~mm}$ (tabl. 1). La proportion de particules de petite taille dans le contenu de feuillet et la répartition des bactéries qui y sont fixées sont vraisemblablement sous-estimées en raison de la faible quantité de phase liquide (riche en petites particules) de l'échantillon prélevé à l'abattage. Sur la base de leur teneur en lipides totaux, les populations bactériennes sont très comparables au niveau du rumen et du feuillet quelle que soit la taille des particules étudiées.

TAB. 1. - Caractéristiques des particules alimentaires et des bactéries fixées sur les particules dans les contenus de rumen (R) et de feuillet (F) chez la vache laitière.

\begin{tabular}{|c|c|c|c|c|c|c|}
\hline \multirow[t]{2}{*}{$\begin{array}{l}\text { Taille des } \\
\text { particules } \\
\text { (en } \mathrm{mm} \text { ) }\end{array}$} & \multicolumn{2}{|c|}{$\begin{array}{c}\text { Répartition des classes } \\
\text { de particules } \\
\text { (en \% MS particules } \\
\text { totales) }\end{array}$} & \multicolumn{2}{|c|}{$\begin{array}{l}\text { Répartition des } \\
\text { bactéries fixées } \\
\text { (en } \% \text { MS bactéries } \\
\text { totales fixées) }\end{array}$} & \multicolumn{2}{|c|}{$\begin{array}{l}\text { Concentration des } \\
\text { bactéries fixées } \\
\text { (en \% MS classe } \\
\text { de particules) }\end{array}$} \\
\hline & $R$ & $F$ & $\mathbf{R}$ & $F$ & $R$ & $F$ \\
\hline $\begin{aligned} & \geq 4 \\
4 & -2 \\
2 & -0,8 \\
0,8 & -0,4 \\
0,4 & -0,1 \\
& <0,1\end{aligned}$ & $\begin{array}{r}51,3 \pm 5,1 \\
2,6 \pm 0,7 \\
8,3 \pm 0,8 \\
5,2 \pm 0,6 \\
8,2 \pm 1,6 \\
24,5 \pm 3,0\end{array}$ & $\begin{array}{r}3,5 \pm 2,5 \\
12,4 \pm 0,9 \\
15,7 \pm 1,8 \\
10,8 \pm 2,5 \\
16,2 \pm 0,8 \\
41,6 \pm 0,5\end{array}$ & $\begin{array}{r}61,6 \pm 8,1 \\
3,7 \pm 1,9 \\
4,9 \pm 1,6 \\
6,4 \pm 4,0 \\
5,1 \pm 1,5 \\
18,4 \pm 5,7\end{array}$ & $\begin{array}{r}1,1 \pm 0,2 \\
13,5 \pm 2,1 \\
14,7 \pm 2,7 \\
7,2 \pm 1,4 \\
12,6 \pm 1,3 \\
52,6 \pm 5,6\end{array}$ & $\begin{array}{l}6,4 \pm 2,1 \\
8,2 \pm 2,8 \\
3,0 \pm 1,0 \\
6,3 \pm 1,4 \\
3,4 \pm 1,3 \\
4,0 \pm 1,6\end{array}$ & $\begin{array}{l}2,6 \pm 1,2 \\
5,9 \pm 0,5 \\
5,2 \pm 1,6 \\
4,0 \pm 1,7 \\
4,3 \pm 0,4 \\
6,8 \pm 0,4\end{array}$ \\
\hline
\end{tabular}

De ce travail, on peut conclure que la réduction en taille des particules alimentaires dans le rumen et le feuillet ne limite pas le taux de fixation des bactéries sur les particules et n'entraîne apparemment pas de sélection particulière parmi les espèces bactériennes fixées. En pratique, l'appréciation du flux duodénal des bactéries fixées pourrait être réalisée en déterminant, au niveau du rumen, la concentration et la composition chimique des bactéries totales fixées aux particules et, au niveau duodénal, le flux total des particules.

Bauchart D., Legay-Carmier F., Doreau M., Jouany J. P., 1986. Reprod. Nutr. Dévelop., 26, 51-52. Merry R. J., Mc Allan A. B., 1983. Br. J. Nutr., 50, 701-709. 